\title{
The link between solenoidal turbulence and slow star formation in G0.253+0.016
}

\author{
C. Federrath ${ }^{1}$, J. M. Rathborne ${ }^{2}$, S. N. Longmore $^{3}$, \\ J. M. D. Kruijssen ${ }^{4,5}$, J. Bally ${ }^{6}$, Y. Contreras ${ }^{7}$, R. M. Crocker ${ }^{1}$, \\ G. Garay ${ }^{8}$, J. M. Jackson ${ }^{9}$, L. Testi ${ }^{10,11,12}$ and A. J. Walsh ${ }^{13}$ \\ ${ }^{1}$ Research School of Astronomy and Astrophysics, Australian National University, Canberra, \\ ACT 2611, Australia \\ email: christoph.federrath@anu.edu.au \\ ${ }^{2}$ CSIRO Astronomy and Space Science, P.O. Box 76, Epping NSW, 1710, Australia \\ ${ }^{3}$ Astrophysics Research Institute, Liverpool John Moores University, IC2, Liverpool Science \\ Park, 146 Brownlow Hill, Liverpool L3 5RF, United Kingdom \\ ${ }^{4}$ Astronomisches Rechen-Institut, Zentrum für Astronomie der Universität Heidelberg, \\ Mönchhofstraße 12-14, 69120 Heidelberg, Germany \\ ${ }^{5}$ Max-Planck Institut für Astronomie, Königstuhl 17, 69117 Heidelberg, Germany \\ ${ }^{6}$ CASA, University of Colorado, 389-UCB, Boulder, CO 80309, USA \\ ${ }^{7}$ Leiden Observatory, Leiden University, PO Box 9513, NL-2300 RA Leiden, the Netherlands \\ ${ }^{8}$ Departamento de Astronomía, Universidad de Chile, Casilla 36-D, Santiago, Chile \\ ${ }^{9}$ Institute for Astrophysical Research, Boston University, Boston, MA 02215, USA \\ ${ }^{10}$ European Southern Observatory, Karl-Schwarzschild-Straße 2, D-85748 Garching bei \\ München, Germany \\ ${ }^{11}$ INAF-Arcetri, Largo E. Fermi 5, I-50125 Firenze, Italy \\ ${ }^{12}$ Excellence Cluster Universe, Boltzmannstraße 2, D-85748, Garching, Germany \\ ${ }^{13}$ International Centre for Radio Astronomy Research, Curtin University, GPO Box U1987, \\ Perth WA 6845, Australia
}

\begin{abstract}
Star formation in the Galactic disc is primarily controlled by gravity, turbulence, and magnetic fields. It is not clear that this also applies to star formation near the Galactic Centre. Here we determine the turbulence and star formation in the CMZ cloud G0.253+0.016. Using maps of $3 \mathrm{~mm}$ dust emission and HNCO intensity-weighted velocity obtained with ALMA, we measure the volume-density variance $\sigma_{\rho / \rho_{0}}=1.3 \pm 0.5$ and turbulent Mach number $\mathcal{M}=11 \pm 3$. Combining these with turbulence simulations to constrain the plasma $\beta=0.34 \pm 0.35$, we reconstruct the turbulence driving parameter $b=0.22 \pm 0.12$ in G0.253+0.016. This low value of $b$ indicates solenoidal (divergence-free) driving of the turbulence in G0.253+0.016. By contrast, typical clouds in the Milky Way disc and spiral arms have a significant compressive (curlfree) driving component $(b>0.4)$. We speculate that shear causes the solenoidal driving in G0.253+0.016 and show that this may reduce the star formation rate by a factor of 7 compared to nearby clouds.
\end{abstract}

Keywords. Galaxy: centre, ISM: clouds, magnetic fields, stars: formation, turbulence

\section{Introduction}

Rathborne et al. $(2014,2015)$ showed that G0.253+0.016 is a dense turbulent cloud in the central molecular zone (CMZ). However, so far it has been unclear what drives this turbulence and whether that turbulence plays a role in controlling the low star formation rate (SFR) seen in G0.253+0.016 and in the CMZ as a whole (Longmore et al. 2013; 


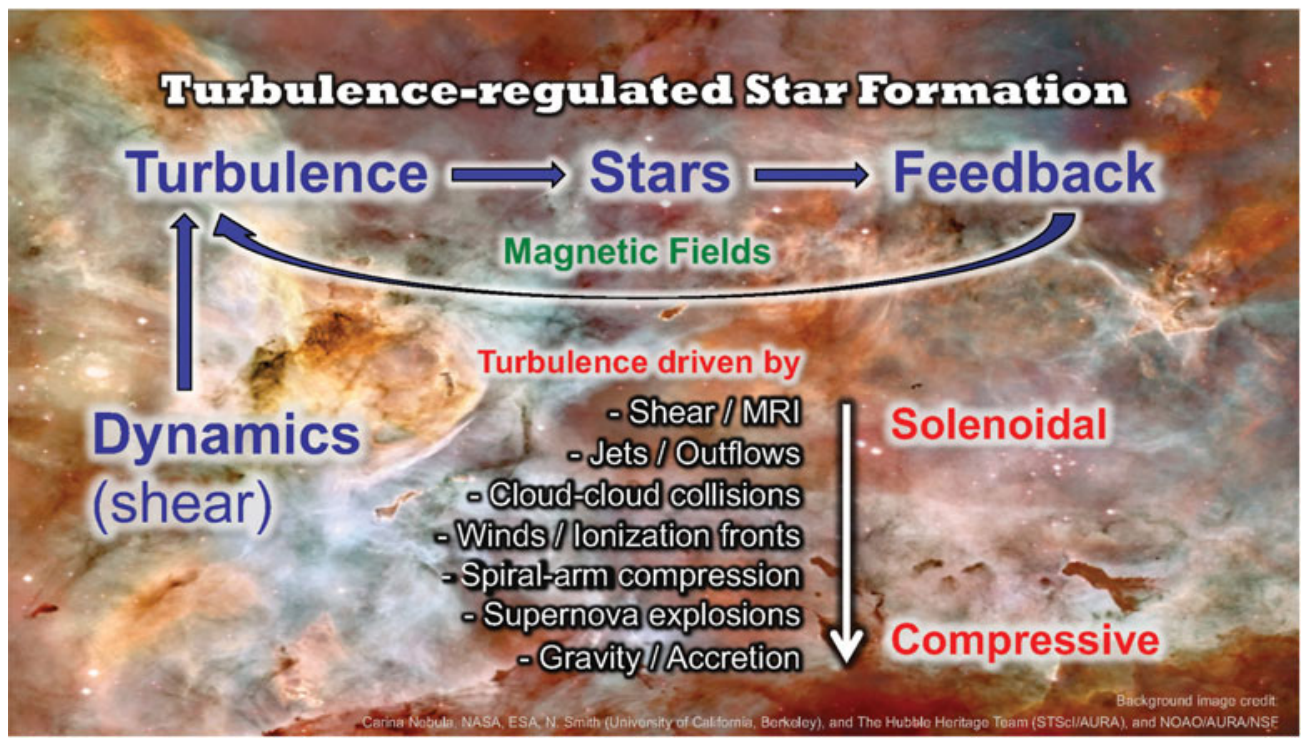

Figure 1. Sketch of the turbulence-regulated paradigm of star formation. Turbulence is fed by stellar feedback and/or large-scale dynamics (such as galactic shear). Different turbulence driving mechanisms can excite more solenoidal (rotational) modes, others inject more compressive (potential) modes. The mix of turbulent modes has profound consequences for star formation.

Kruijssen et al. 2014; Johnston et al. 2014). Using high-resolution ALMA $3 \mathrm{~mm}$ dust and HNCO molecular line data, we determine the driving mode of the turbulence in G0.253+0.016 and link the turbulence driving to the SFR.

The turbulence-regulated paradigm of star formation (Mac Low \& Klessen 2004; Elmegreen \& Scalo 2004; McKee \& Ostriker 2007; Hennebelle \& Falgarone 2012; Federrath \& Klessen 2012; Padoan et al. 2014) provides us with the basic framework for our approach to determine the turbulence parameters of G0.253+0.016 and allows us to make predictions for the star formation activity in G0.253+0.016. Figure 1 shows a sketch of the turbulence-regulated picture of star formation. In this model, turbulence shapes the density distribution of the clouds, thereby controlling the dense-gas fraction and thus, the formation of stars. Then, stellar feedback (such as supernova explosions or stellar winds) and/or large-scale dynamics (such as galactic shear or magneto-rotational instability) drive the turbulence. Understanding and determining the drivers of the turbulence is of fundamental importance in this model of star formation.

Idealised numerical simulations have shown that compressible, supersonic turbulence decays quickly, in about a crossing time (Scalo \& Pumphrey 1982; Mac Low et al. 1998; Stone et al. 1998; Mac Low 1999). Given that G0.253+0.016 and other galactic clouds are in a dynamic state of supersonic turbulence means that the turbulence is driven by some physical stirring mechanism(s).

Turbulence driving mechanisms can be broadly separated into two groups: 1) stellar feedback, and 2) gas dynamics caused by mechanisms other than feedback. Stellar feedback includes supernova explosions, stellar winds, and ionisation fronts (McKee 1989; Krumholz et al. 2006; Balsara et al. 2004; de Avillez \& Breitschwerdt 2005; Breitschwerdt et al. 2009; Gritschneder et al. 2009; Peters et al. 2010, 2011; Arce et al. 2011; Goldbaum et al. 2011; Lee et al. 2012), primarily caused by high-mass stars, as well as jets and outflows from young stars, including low- and intermediate-mass stars (Norman \& Silk 1980; Matzner \& McKee 2000; Banerjee et al. 2007; Nakamura \& Li 2008; Cunningham 
et al. 2009; Carroll et al. 2010; Wang et al. 2010; Cunningham et al. 2011; Plunkett et al. 2013, 2015; Offner \& Arce 2014; Federrath et al. 2014). The 2nd category (which we refer to as "Dynamics" in Figure 1) includes accretion (such as accretion onto a galaxy) and gravitational collapse (Hoyle 1953; Vazquez-Semadeni et al. 1998; Klessen \& Hennebelle 2010; Elmegreen \& Burkert 2010; Vázquez-Semadeni et al. 2010; Federrath et al. 2011; Robertson \& Goldreich 2012; Lee et al. 2015), the magneto-rotational instability (MRI) (Balbus \& Hawley 1991; Piontek \& Ostriker 2004, 2007; Tamburro et al. 2009), spiral-arm compression (Dobbs \& Bonnell 2008; Dobbs et al. 2008), cloud-cloud collisions (Tasker \& Tan 2009; Benincasa et al. 2013), and shear. While different drivers can play a role in different environments, Kruijssen et al. (2014) found that most of these drivers are not sufficient to explain the turbulent velocity dispersion in the CMZ, but some of them can.

A critical consideration is that the majority of turbulence drivers (e.g., supernova explosions, high-mass stellar winds, and accretion) primarily drive compressible (curl-free) modes, so we refer to these as "compressive drivers". By contrast, solenoidal (divergencefree) modes can be generated directly by shear and the MRI (so we call them "solenoidal drivers"). The key aspect here is that the density probability distribution function (PDF) depends critically on the driving. Federrath et al. (2008, 2010); Price et al. (2011); Molina et al. (2012); Konstandin et al. (2012); Nolan et al. (2015); Federrath \& Banerjee (2015) showed that the variance (width) of the density PDF is given by

$$
\sigma_{\rho / \rho_{0}}=b \mathcal{M}\left(1+\beta^{-1}\right)^{-1 / 2},
$$

with the turbulent Mach number $\mathcal{M}=\sigma_{v} / c_{\mathrm{s}}$ (the ratio of velocity dispersion and sound speed), plasma $\beta$ (the ratio of thermal and magnetic pressure), and the turbulence driving parameter $b$, which smoothly varies from $b=1 / 3$ for purely solenoidal driving to $b=1$ for purely compressive driving (Federrath et al. 2010).

The theoretical models and simulations in Federrath \& Klessen (2012) demonstrated that the SFR depends on $b$, with compressive driving producing up to an order of magnitude higher SFRs than solenoidal driving. Thus, our goal is to determine whether the driving of turbulence in G0.253+0.016 is primarily solenoidal or compressive. We do this by measuring $\sigma_{\rho / \rho_{0}}, \mathcal{M}$, and $\beta$, and inverting Equation (1.1) to solve for $b$. Finally, we use our measurement of $b$ to predict the SFR in G0.253+0.016 and to contrast this to the SFR in Milky Way clouds located in the Galactic disc rather than the Galactic Centre.

\section{Results}

The main results and methods of this work are published in Federrath et al. (2016). Here we summarise the main results concerning the driving mode of the turbulence in G0.253+0.016 and its implications for the SFR.

Figure 2 shows the $\mathrm{H}_{2}$ column density and the $\mathrm{HNCO}$ intensity-weighted velocity in G0.253+0.016. In order to isolate primarily turbulent motions in the cloud, we have subtracted the large-scale velocity gradient seen across G0.253+0.016 (Rathborne et al. 2015). From the two maps in Figure 2, we compute the column density PDF and the turbulent velocity PDF shown in Figure 3, respectively. We find $\sigma_{\eta}=0.34 \pm 0.02$ (as reported by Rathborne et al. 2014) for the log-normalised column density contrast $\eta=$ $\ln (N /\langle N\rangle)$ and the $1 \mathrm{D}$ velocity dispersion $\sigma_{v, 1 \mathrm{D}}=3.9 \pm 0.1 \mathrm{~km} \mathrm{~s}^{-1}$, both in the plane of the sky. Using temperature measurements from Ginsburg et al. (2016), we find sound speeds $c_{\mathrm{s}}=0.60 \pm 0.15 \mathrm{~km} \mathrm{~s}^{-1}$, leading to the $3 \mathrm{D}$ turbulent Mach number, $\mathcal{M}=11 \pm 3$ in G0.253+0.016.

In order to apply Equation (1.1) to solve for the turbulence driving parameter $b$, we 

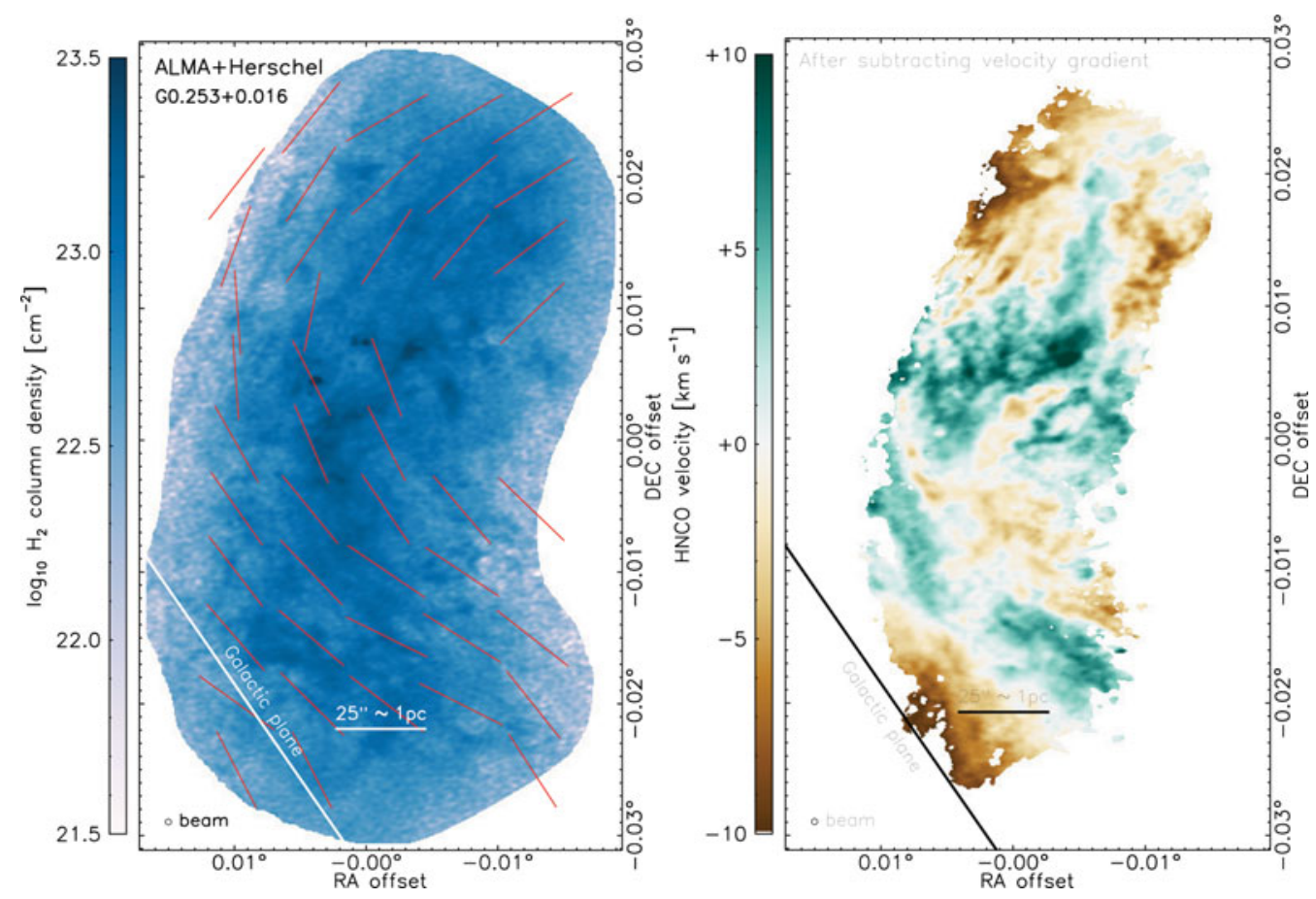

Figure 2. Left-hand panel: $\mathrm{H}_{2}$ column density map of G0.253+0.016 from combined ALMA+Herschel data (Rathborne et al. 2014). The direction of the large-scale magnetic field from polarisation measurements obtained in Dotson et al. (2010) and Pillai et al. (2015) is shown as red pseudo vectors. Right-hand panel: HNCO intensity-weighted velocity of G0.253+0.016 after subtraction of the large-scale velocity gradient across the cloud. The gradient-subtracted map primarily depicts the turbulent motions in the plane of the sky. Both maps are in equatorial coordinates with the $(0,0)$ offset position in right ascension (RA) and declination (DEC) being 17:46:09.59, -28:42:34.2 J2000, respectively.

need to convert the column-density variance $\sigma_{\eta}$ to the volume-density variance $\sigma_{\rho / \rho_{0}}$. We use the technique developed in Brunt et al. (2010b,a) (see also Kainulainen et al. 2014) and find $\sigma_{\rho / \rho_{0}}=1.3 \pm 0.5$. Finally, we need an estimate for the turbulent (unordered) magnetic-field plasma $\beta$ parameter. We use the ordered magnetic field measurement from Pillai et al. (2015) (see polarisation pseudo vectors in Figure 2) and run magnetohydrodynamical turbulence simulations with this ordered field and the measured $\mathcal{M}$. From the simulations, we determine the unordered, turbulent plasma $\beta=0.34 \pm 0.35$ entering Equation (1.1).

Combining our measurements of $\sigma_{\rho / \rho_{0}}, \mathcal{M}$, and $\beta$ and propagating the uncertainties, we find $b=0.22 \pm 0.12$ in G0.253+0.016, indicating solenoidal driving of the turbulence. We interpret this driving to be the result of strong shearing motions in the CMZ (Krumholz \& Kruijssen 2015, Kruijssen et al., in prep.). This is in stark contrast to the driving parameter inferred for typical clouds in the Galactic disc and spiral arms of the Milky Way. Currently available measurements for Taurus (Brunt 2010), GRSMC43.300.33 (Ginsburg et al. 2013) and IC5146 (Padoan et al. 1997) all show $b>0.4$ with typical values of $b=0.5$, thus exhibiting a significant compressive driving component, in contrast to the CMZ cloud G0.253+0.016. 

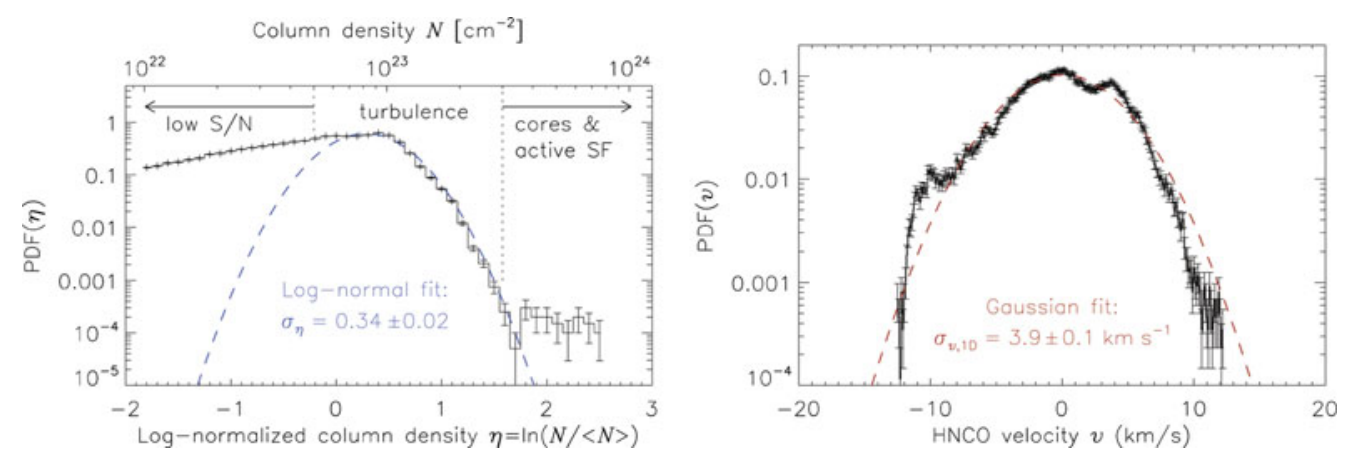

Figure 3. Left-hand panel: column density PDF of the map shown in the left-hand panel of Figure 2, for the log-normalised column density contrast $\eta=\ln (N /\langle N\rangle)$. A log-normal fit (dashed line) in the turbulence-dominated regime gives $\sigma_{\eta}=0.34 \pm 0.02$ (Rathborne et al. 2014). Right-hand panel: HNCO velocity PDF of the map shown in the right-hand panel of Figure 2 . From a Gaussian fit, we measure the 1D turbulent velocity dispersion $\sigma_{v, 1 \mathrm{D}}=3.9 \pm 0.1 \mathrm{~km} \mathrm{~s}^{-1}$.

\section{Implications for the SFR}

Using our measurements of $b, \mathcal{M}$, and $\beta$, we find a predicted $\mathrm{SFR}=(1.1 \pm 0.8) \times$ $10^{-2} M_{\odot} \mathrm{yr}^{-1}$, based on the theoretical multi-freefall models of the SFR summarised in Federrath \& Klessen (2012). If we used a turbulence driving parameter $b=0.5$ (as measured for clouds in the Galactic disc and spiral arms), we would find a 7 times higher SFR. This shows that the driving of the turbulence is a critical parameter for star formation and may significantly contribute to explaining the low SFRs in G0.253+0.016 and possibly in the entire CMZ.

\section{References}

Arce, H. G., Borkin, M. A., Goodman, A. A., Pineda, J. E., \& Beaumont, C. N. 2011, ApJ, 742, 105

Balbus, S. A. \& Hawley, J. F. 1991, ApJ, 376, 214

Balsara, D. S., Kim, J., Mac Low, M., \& Mathews, G. J. 2004, ApJ, 617, 339

Banerjee, R., Klessen, R. S., \& Fendt, C. 2007, ApJ, 668, 1028

Benincasa, S. M., Tasker, E. J., Pudritz, R. E., \& Wadsley, J. 2013, ApJ, 776, 23

Breitschwerdt, D., de Avillez, M. A., Fuchs, B., \& Dettbarn, C. 2009, SSRv, 143, 263

Brunt, C. M. 2010, A\&A, 513, A67

Brunt, C. M., Federrath, C., \& Price, D. J. 2010a, MNRAS, 405, L56

-. 2010b, MNRAS, 403, 1507

Carroll, J. J., Frank, A., \& Blackman, E. G. 2010, ApJ, 722, 145

Cunningham, A. J., Frank, A., Carroll, J., Blackman, E. G., \& Quillen, A. C. 2009, ApJ, 692, 816

Cunningham, A. J., Klein, R. I., Krumholz, M. R., \& McKee, C. F. 2011, ApJ, 740, 107

de Avillez, M. A. \& Breitschwerdt, D. 2005, A\&A, 436, 585

Dobbs, C. L. \& Bonnell, I. A. 2008, MNRAS, 385, 1893

Dobbs, C. L., Glover, S. C. O., Clark, P. C., \& Klessen, R. S. 2008, MNRAS, 389, 1097

Dotson, J. L., Vaillancourt, J. E., Kirby, L., et al. 2010, ApJ, 186, 406

Elmegreen, B. G. \& Burkert, A. 2010, ApJ, 712, 294

Elmegreen, B. G. \& Scalo, J. 2004, ARAA, 42, 211

Federrath, C. \& Banerjee, S. 2015, MNRAS, 448, 3297

Federrath, C. \& Klessen, R. S. 2012, ApJ, 761, 156

Federrath, C., Klessen, R. S., \& Schmidt, W. 2008, ApJ, 688, L79

Federrath, C., Roman-Duval, J., Klessen, R. S., Schmidt, W., \& Mac Low, M. 2010, A\&A, 512, A 81 
Federrath, C., Schrön, M., Banerjee, R., \& Klessen, R. S. 2014, ApJ, 790, 128

Federrath, C., Sur, S., Schleicher, D. R. G., Banerjee, R., \& Klessen, R. S. 2011, ApJ, 731, 62

Federrath, C., Rathborne, J. M., Longmore, S. N., et al. 2016, ApJ, accepted (arXiv:1609.05911)

Ginsburg, A., Federrath, C., \& Darling, J. 2013, ApJ, 779, 50

Ginsburg, A., Henkel, C., Ao, Y., et al. 2016, A\&A, 586, A50

Goldbaum, N. J., Krumholz, M. R., Matzner, C. D., \& McKee, C. F. 2011, ApJ, 738, 101

Gritschneder, M., Naab, T., Walch, S., Burkert, A., \& Heitsch, F. 2009, ApJ, 694, L26

Hennebelle, P. \& Falgarone, E. 2012, A\&A Rev., 20, 55

Hoyle, F. 1953, ApJ, 118, 513

Johnston, K. G., Beuther, H., Linz, H., et al. 2014, A\&A, 568, A56

Kainulainen, J., Federrath, C., \& Henning, T. 2014, Science, 344, 183

Klessen, R. S. \& Hennebelle, P. 2010, A\&A, 520, A17

Konstandin, L., Girichidis, P., Federrath, C., \& Klessen, R. S. 2012, ApJ, 761, 149

Kruijssen, J. M. D., Longmore, S. N., Elmegreen, B. G., et al. 2014, MNRAS, 440, 3370

Krumholz, M. R. \& Kruijssen, J. M. D. 2015, MNRAS, 453, 739

Krumholz, M. R., Matzner, C. D., \& McKee, C. F. 2006, ApJ, 653, 361

Lee, E. J., Chang, P., \& Murray, N. 2015, ApJ, 800, 49

Lee, E. J., Murray, N., \& Rahman, M. 2012, ApJ, 752, 146

Longmore, S. N., Bally, J., Testi, L., et al. 2013, MNRAS, 429, 987

Mac Low, M.-M. 1999, ApJ, 524, 169

Mac Low, M.-M. \& Klessen, R. S. 2004, RvMP, 76, 125

Mac Low, M.-M., Klessen, R. S., Burkert, A., \& Smith, M. D. 1998, PhRvL, 80, 2754

Matzner, C. D. \& McKee, C. F. 2000, ApJ, 545, 364

McKee, C. F. 1989, ApJ, 345, 782

McKee, C. F. \& Ostriker, E. C. 2007, ARAA, 45, 565

Molina, F. Z., Glover, S. C. O., Federrath, C., \& Klessen, R. S. 2012, MNRAS, 423, 2680

Nakamura, F. \& Li, Z. 2008, ApJ, 687, 354

Nolan, C. A., Federrath, C., \& Sutherland, R. S. 2015, MNRAS, 451, 1380

Norman, C. \& Silk, J. 1980, ApJ, 238, 158

Offner, S. S. R. \& Arce, H. G. 2014, ApJ, 784, 61

Padoan, P., Federrath, C., Chabrier, G., et al. 2014, Protostars and Planets VI, 77

Padoan, P., Jones, B. J. T., \& Nordlund, A. P. 1997, ApJ, 474, 730

Peters, T., Banerjee, R., Klessen, R. S., \& Mac Low, M. 2011, ApJ, 729, 72

Peters, T., Banerjee, R., Klessen, R. S., et al. 2010, ApJ, 711, 1017

Pillai, T., Kauffmann, J., Tan, J. C., et al. 2015, ApJ, 799, 74

Piontek, R. A. \& Ostriker, E. C. 2004, ApJ, 601, 905

-. 2007, ApJ, 663, 183

Plunkett, A. L., Arce, H. G., Corder, S. A., et al. 2015, ApJ, 803, 22

—. 2013, ApJ, 774, 22

Price, D. J., Federrath, C., \& Brunt, C. M. 2011, ApJ, 727, L21

Rathborne, J. M., Longmore, S. N., Jackson, J. M., et al. 2014, ApJ, 795, L25

-. 2015, ApJ, 802, 125

Robertson, B. \& Goldreich, P. 2012, ApJ, 750, L31

Scalo, J. M. \& Pumphrey, W. A. 1982, ApJ, 258, L29

Stone, J. M., Ostriker, E. C., \& Gammie, C. F. 1998, ApJ, 508, L99

Tamburro, D., Rix, H.-W., Leroy, A. K., et al. 2009, AJ, 137, 4424

Tasker, E. J. \& Tan, J. C. 2009, ApJ, 700, 358

Vazquez-Semadeni, E., Canto, J., \& Lizano, S. 1998, ApJ, 492, 596

Vázquez-Semadeni, E., Colín, P., Gómez, G. C., Ballesteros-Paredes, J., \& Watson, A. W. 2010, ApJ, 715, 1302

Wang, P., Li, Z.-Y., Abel, T., \& Nakamura, F. 2010, ApJ, 709, 27 